Pacific

Journal of

Mathematics

APPLICATION OF REPRESENTATION FORMULAE TO COMPARISON AND NONEXISTENCE THEOREMS FOR ELLIPTIC BOUNDARY VALUE PROBLEMS

A.A. Kosmodem'yanskiI JR. 


\title{
APPLICATION OF REPRESENTATION FORMULAE TO COMPARISON AND NONEXISTENCE THEOREMS FOR ELLIPTIC BOUNDARY VALUE PROBLEMS
}

\author{
A.A. Kosmodem'yanskit JR.
}

Using the representation formulas obtained earlier, new comparison theorems for elliptic boundary value problems are developed. Properties of support function of convex domain are applied for proofs and for obtaining nonexistence theorems for solutions of capillary problems in the absence of gravity.

Let $D_{0}$ and $D_{1} \quad\left(D_{0} \subset D_{1}\right)$ be plane convex figures. Denote by $A_{i}$ and $p_{i}$ the area and the perimeter of figure $D_{i} \quad(i=0,1)$. Let's suppose that the inequality

$$
\frac{A_{1}}{p_{1}}>\frac{A_{0}}{p_{0}}
$$

holds. In the present paper, using (1), we shall obtain the comparison theorems for some elliptic boundary value problems (Sections 1-3). Proofs of these theorems were based on representation formulas, obtained earlier. The general steps of proving one of these are given in the Appendix. Further (Section 4) we will formulate the sufficient condition for (1) in terms of mixed area and will use these results to prove some nonexistence theorems for solutions of capillary problems in the absence of gravity (Section 5).

\section{The comparison theorem for solutions of second boundary} value problem for Helmholtz equation.

Let $D$ be a convex planar domain with $C^{2, \alpha}$ boundary $\Gamma$. Hereinafter we denote by $n$ the outward normal to $\Gamma$. Let $u(x, y)$ be a solution to the following problem

$$
\Delta u=k u \quad \text { in } D,\left.\quad u_{n}\right|_{\Gamma}=R>0 .
$$

In [7] we have proved the following:

Theorem 1. Let $u$ be the solution to problem (2), and $z$ be a solution of second boundary value problem for the Poisson equation

$$
\Delta z=\frac{R p}{A} \quad \text { in } D,\left.\quad z_{n}\right|_{\Gamma}=R,
$$


such that

$$
\iint_{D} z d x d y=0 .
$$

Then the solution of problem (2) can be represented as

$$
u=\frac{R p}{k A}+z+\omega
$$

where $\omega$ satisfies the inequality $\max |\omega|<C|k|$ in $D$.

Let $u_{i}$ be the solution to (2) in the domain $D_{i}$. From (4) we immediately obtain:

Theorem 2. Let domains $D_{0}$ and $D_{1}$ be such that the conditions of Theorem 1 and inequality (1) hold. Then there exists a number $k_{0}>0$ such that for any positive number $k<k_{0}$ the inequality $u_{0}>u_{1}$ holds in the domain $D_{0}$.

The result of Theorem 2 we had anounced in $[6]$.

\section{The comparison theorem for solutions of the third boundary value problem for Poisson equation.}

In the third boundary value problem it is required to find a solution of the equation

$$
\Delta u=-1
$$

in domain $D$ with the boundary conditions

$$
u+\beta \frac{\partial u}{\partial n}=0 \quad(\beta>0) .
$$

The solution of this problem satisfies the theorem of representation ([5], [8]).

Theorem 3. Let the boundary $\Gamma$ of plane convex domain $D$ belongs to the class $C^{2, \alpha}$ and its curvature is separated from zero. Then

$$
u=\frac{\beta A}{p}+u_{\infty}+\omega
$$

where $u_{\infty}$ is the solution of Equation (5) such that

$$
\frac{\partial u_{\infty}}{\partial n}=-\frac{A}{p}, \quad \int_{\Gamma} u_{\infty} d s=0,
$$

and function $\omega$ satisfies the inequality $\max |\omega|<C \beta^{-1}$ in $D$.

Let $u_{i}$ be the solutions of the third boundary value problems in domains $D_{i}$. From representation (7) we immediately obtain: 
Theorem 4. Let the domains $D_{0}$ and $D_{1}$ be such that the conditions of Theorem 3 and inequality (1) hold. Then there exists $\beta_{0}>0$ such that for any $\beta>\beta_{0}$ the inequality $u_{1}>u_{0}$ holds in $D_{0}$.

\section{The comparison theorem of capillary surfaces heights in case of small gravity.}

It is well-known (see [3]) that the searching of the form of liquid free surface in cylindrical tube under capillary forces and force of gravity is equivalent to the following boundary value problem. It is required to find the solution of the equation

$$
\operatorname{div} T u=k u
$$

in domain $D$ with boundary condition

$$
(T u, n)=\cos \gamma, \quad\left(T u=\nabla u / \sqrt{1+|\nabla u|^{2}}\right) .
$$

In the absence of gravity, the equation of liquid free surface takes the form

$$
\operatorname{div} T u=\frac{p \cos \gamma}{A},
$$

but boundary condition remains in form (9).

Below we consider M. Miranda question ([3], Sec. 5.3, 5.4): Does a liquid in a "wide" capillary tube rise lower than in a "narrow" one. This question is equivalent to the following problem: Let $u_{0}$ and $u_{1}$ be solutions of Equation (8) in domains $D_{0}$ and $D_{1} \quad\left(D_{0} \subset D_{1}\right)$ with boundary conditions (9) on boundaries $\Gamma_{0}$ and $\Gamma_{1}$. Is it right that $u_{0}>u_{1}$ in $D_{0}$ ?

In [3] some conditions for an affirmative answer are given, and also an example for which the answer is negative.

D. Siegel has proved in [13] for plane domain with $C^{2, \alpha}$ boundary the following:

Theorem 5. Let there exists a solution $z$ to the problem (10)-(9). Then solution $u$ of the problem (8)-(9) can be represented as

$$
u=\frac{p \cos \gamma}{k A}+z+\omega
$$

while the function $\omega$ satisfies the inequality $\max |\omega|<C|k|$ in $D$.

$L_{2}$-estimate of $\omega$ was received in [7].

Now the comparison theorem is immediately following from representation (11).

Theorem 6. Let $0<\gamma<\pi / 2$, the inequality (1) holds and there exist the solutions of the problem (10)-(9) in domains $D_{0}$ and $D_{1}$, then exists $k_{0}>0$ such that for any $0<k<k_{0}$ the inequality $u_{0}>u_{1}$ holds in $D_{0}$. 
We note, that for special cases of domains $D_{i}\left(D_{1}\right.$ is a disk or $D_{0}$ is a disk of sufficiently small radius), the comparison Theorems 2,4 and 6 have been obtained by other methods for arbitrary positive $k$ and $\beta$ in [3], [12], [5].

On the other hand, it is evident that if the inequality reverse (1) holds, then $u_{0}<u_{1}$ in $D_{0}$.

\section{Geometrical theorem.}

Let us obtain now a sufficient condition under which the inequality (1) holds. We have proved the same implication in [6], where we assume sufficient smoothness of a boundaries. In present paper this result is reduced in Example 3 .

Let $A_{01}$ be the mixed area of figures $D_{0}$ and $D_{1}$.

Theorem 7. Let figures $D_{0}$ and $D_{1}$ be such that

$$
\left(p_{0}+p_{1}\right) A_{1} \geq 2 A_{01} p_{1} .
$$

Then the inequality (1) holds.

Proof. We shall use the formulas from standard manuals $([\mathbf{1}],[\mathbf{1 1}])$ on the geometry of convex figures.

Let $D_{\theta}=(1-\theta) D_{0}+\theta D_{1}$ be the linear family of convex figures. It is well-known that the area $A_{\theta}$ of the figure $D_{\theta}$ is given by formula

$$
A_{\theta}=(1-\theta)^{2} A_{0}+2 \theta(1-\theta) A_{01}+\theta^{2} A_{1},
$$

and its perimeter $p_{\theta}$ is given by formula

$$
p_{\theta}=(1-\theta) p_{0}+\theta p_{1} .
$$

We note that inequality (1) immediately follows from (12) and the Frobenius inequality

$$
2 A_{01} \geq \frac{A_{0} p_{1}}{p_{0}}+\frac{A_{1} p_{0}}{p_{1}} .
$$

We shall give another proof whose details give additional information. Let us consider the function

$$
f(\theta)=\frac{A_{\theta}}{p_{\theta}} .
$$

We shall prove that this function is concave in the segment $[0,1]$ and its left derivative $f^{\prime}(1)$ is positive because of (12). Hence we shall prove that the function $f(\theta)$ monotonically increases. Using formulas (13) and (14), we obtain

On the other hand

$$
f(\theta)=\frac{(1-\theta)^{2} A_{0}+2 \theta(1-\theta) A_{01}+\theta^{2} A_{1}}{(1-\theta) p_{0}+\theta p_{1}} .
$$

$$
(1-\theta) f(0)+\theta f(1)=(1-\theta) \frac{A_{0}}{p_{0}}+\theta \frac{A_{1}}{p_{1}} .
$$


After elementary algebraic transformations we see that the concavity condition for $f(\theta)$

$$
f(\theta) \geq(1-\theta) f(0)+\theta f(1)
$$

is equivalent to Frobenius inequality. If we calculate the left derivative

$$
f^{\prime}(1)=\lim _{\epsilon \rightarrow 0} \frac{f(1)-f(1-\epsilon)}{\epsilon},
$$

using formulas (13) and (14), we obtain

$$
\begin{aligned}
f(1)-f(1-\epsilon) & =\frac{F_{1}}{p_{1}}-\frac{\epsilon^{2} F_{0}+2 \epsilon(1-\epsilon) F_{01}+(1-\epsilon)^{2} F_{1}}{\epsilon p_{0}+(1-\epsilon) p_{1}} \\
& =\epsilon \frac{\left(p_{0}+p_{1}\right) A_{1}-2 A_{01} p_{1}}{p_{1}^{2}}+O\left(\epsilon^{2}\right) .
\end{aligned}
$$

It is evident that the derivative $f^{\prime}(1)$ (the coefficient of $\epsilon$ in (15)) is nonnegative because of (12).

Theorem 7 has been proved.

If coefficient of $\epsilon$ in (15) is negative then inequality opposite (12) holds. This means that between figures $D_{\theta}$ there exists the figure such that $f(\theta)>$ $f(1)$.

On the other hand we shall obtain the condition for inequality opposite (1) if we calculate the right derivative of the function $f(\theta)$ in zero.

Theorem 8. Let

Then

$$
2 A_{01} \leq \frac{p_{0}+p_{1}}{p_{0}} A_{0} .
$$

$$
\frac{A_{1}}{p_{1}}<\frac{A_{0}}{p_{0}} .
$$

Let us consider three important special cases.

Example 1. Let $D_{1}$ be a disk with radius $R_{1}$. In this case we have $2 A_{01}=$ $R_{1} p_{0}$. It is evident that $p_{0}<2 \pi R_{1}$, hence

$$
R_{1} p_{0} 2 \pi R_{1}<\left(p_{0}+2 \pi R_{1}\right) \pi R_{1}^{2},
$$

and inequality (12) holds.

Example 2. Let $D_{0}$ be a disk with radius

$$
r<\frac{A_{1}}{p_{1}} \frac{1}{1-\frac{2 \pi A_{1}}{p_{1}^{2}}} .
$$

We know that $2 A_{01}=r p_{1}$. After algebraic transformations we obtain

$$
r\left(p_{1}^{2}-2 \pi A_{1}\right)<A_{1} p_{1},
$$

or

$$
r p_{1} p_{1}<\left(2 \pi r+p_{1}\right) A_{1},
$$


hence inequality (12) holds.

We note that from isoperimetric inequality

$$
\frac{A_{1}}{p_{1}} \frac{1}{1-\frac{2 \pi A_{1}}{p_{1}^{2}}} \leq \frac{2 A_{1}}{p_{1}}
$$

(equality holds only if $D_{1}$ is a disk), hence, using Example 1, we can to improve the previous result: Let $D_{0} \subset D_{1}$ and $D_{0}$ is contained in the disc of radius $R_{0}<2 A_{1} / p_{1}$ then inequality (1) holds.

Example 3. Let domain $D_{1}$ has smooth boundary, whose curvature $K_{1}$ satisfies the inequality

$$
0<K_{1} \leq \frac{p_{1}}{A_{1}}
$$

Then inequality (12) holds.

Indeed, let $h_{i}(\phi)$ be the support function of the domain $D_{i}$. Then the following formulas are valid ([1])

$$
\begin{gathered}
2 A_{01}=\int_{0}^{2 \pi}\left(h_{0} h_{1}-h_{0}^{\prime} h_{1}^{\prime}\right) d \phi, \\
\frac{1}{K_{1}}=h_{1}^{\prime \prime}+h_{1}, \\
p=\int_{0}^{2 \pi} h d \phi, \quad 2 A=\int_{0}^{2 \pi}\left(h^{2}-h^{\prime 2}\right) d \phi .
\end{gathered}
$$

Using the inequality (16) we obtain

$$
\begin{aligned}
2 A_{01} & =\int_{0}^{2 \pi} h_{0}\left(h_{1}^{\prime \prime}+h_{1}\right) d \phi \\
& =\int_{0}^{2 \pi}\left(h_{0}-h_{1}\right)\left(h_{1}^{\prime \prime}+h_{1}\right) d \phi+\int_{0}^{2 \pi}\left(h_{1}^{2}-h_{1}^{\prime 2}\right) d \phi \\
& \leq 2 A_{1}-\frac{A_{1}}{p_{1}}\left(p_{1}-p_{0}\right) \\
& =\frac{A_{1}\left(p_{1}+p_{0}\right)}{p_{1}} .
\end{aligned}
$$

Hence the inequality (12) holds.

Examples stated above show that condition (12) can be used for checking inequality (1). 


\section{The nonexistence theorems for solutions of capillary problem in the absence of gravity.}

Let us return to the problem (10)-(9). If $\gamma=0$ the important condition of the existence of solution for this problem in the domain $D_{1}$ is the following: Let $D_{0}$ be an arbitrary subdomain of $D_{1}$ then if the solution of the problem (9)-(10) exists then inequality (1) holds. ([2]).

Giusti has proved ([4]) that for convex domains the sufficient condition of the existence is the inequality (16). Moreover $([3])$, if the solution of the problem (10)-(9) exists for $\gamma=0$ then it exists for any $0<\gamma \leq \pi / 2$. Using this statements and our previous speculations we can reformulate the result [2] as sufficient condition of nonexistence for problem (10)-(9).

Theorem 9. Let exists such convex subdomain $D_{0}$ of domain $D_{1}$ that the inequality opposite (12) holds. Then if $\gamma=0$ then a solution of (10)-(9) does not exist.

Proof. Really, if inequality opposite (12) holds then there exists a domain $\bar{D} \subset D_{1}$ in linear family $D_{\theta}=(1-\theta) D_{0}+\theta D_{1}$ such that

$$
\frac{\bar{A}}{\bar{p}}>\frac{A_{1}}{p_{1}} .
$$

In particular, (10)-(9) has no solutions, if $D_{1}$ is a regular polygon. Indeed, we can put as $D_{0}$ the disk inscribed into $D_{1}$.

Using results of Section 4, we can add the following simple condition of nonexistence of solutions for (10)-(9) in case of $\gamma=0$.

Theorem 10. Let we can inscribe into $D_{1}$ the disk of radius

$$
r>\frac{p_{1} A_{1}}{p_{1}^{2}-2 \pi A_{1}} .
$$

Then in case of $\gamma=0$ the solution of the problem (10)-(9) does not exist.

Proof. We immediately obtain from (17)

$$
r p_{1}>\frac{2 \pi r+p_{1}}{p_{1}} A_{1} \text {. }
$$

We can take a disk of radius $r$ as the domain $D_{0}$. It is evident that the inequality (18) is the inequality opposite (12), hence we can apply Theorem 9.

Let us obtain now the generalization of Theorem 10 .

Theorem 11. Let we can inscribe in domain $D_{1}$ the disc of radius $r$ such that inequality (17) holds. Then problem (10)-(9) has no solutions for any contact angle $\gamma$ satisfying the inequality

$$
\cos \gamma>\frac{A_{1}}{r p_{1}}\left(1+\sqrt{\frac{4 \pi\left(p_{1} r-\pi r^{2}-A_{1}\right)}{p_{1}^{2}-4 \pi A_{1}}}\right) .
$$


Proof. We remind the general idea of nonexistence proofs: If we can find subdomain $\bar{D} \subset D_{1}$ such that

$$
\frac{A_{1}}{p_{1}}<\frac{\bar{A} \cos \gamma}{\bar{p}}
$$

then problem (10)-(9) has no solution ([3]).

We shall find the subdomain $\bar{D}$ in a certain linear family $D_{\theta}$. We can reformulate the nonexistence condition in the following form: Let there exist a subdomain $D_{0} \subset D_{1}$ and number $\bar{\theta} \in(0,1)$ such that

$$
f(1)<f(\bar{\theta}),
$$

then the problem (10)-(9) has no solutions.

Let us construct the corresponding linear family.

Let the convex domain $D_{0} \subset D_{1}$ be such that

$$
2 A_{01} p_{0}>\left(p_{0}+p_{1}\right) A_{0}, \quad 2 A_{01} p_{1}>\left(p_{0}+p_{1}\right) A_{1} .
$$

It follows from Theorems 7 and 8 , that function $f(\theta)$ reaches its maximum value in the interval $(0,1)$. Let us find this value. We represent the function $f(\theta)$ in the form

$$
f(\theta)=-\frac{E \theta}{\Delta p}+\frac{S}{(\Delta p)^{2}}-\frac{G}{(\Delta p)^{2}\left(\theta \Delta p+p_{0}\right)},
$$

where

$$
\begin{gathered}
E=2 A_{01}-A_{0}-A_{1}, \quad S=2 p_{1}\left(A_{01}-A_{0}\right)+p_{0}\left(A_{0}-A_{1}\right), \\
G=2 p_{0} p_{1} A_{01}-p_{1}^{2} A_{0}-p_{0}^{2} A_{1}, \quad \Delta p=p_{1}-p_{0} .
\end{gathered}
$$

It follows from the Frobenius inequality that $G>0$, and inequalities (22) shows that $E>0$.

After calculations we see that function $f(\theta)$ reaches its maximum value in the point

$$
\bar{\theta}=\frac{1}{\Delta p}\left(\sqrt{\frac{G}{E}}-p_{0}\right),
$$

and this value is equal to

$$
f(\bar{\theta})=\frac{2}{(\Delta p)^{2}}\left(\left(p_{0}+p_{1}\right) A_{01}-p_{1} A_{0}-p_{0} A_{1}-\sqrt{G E}\right) .
$$

Using inequality (21), we can reformulate the sufficient condition of nonexistence of solution to the problem (10)-(9): Let there exist a convex subdomain $D_{0}$ of the domain $D_{1}$ such that inequalities (22) holds, then for any contact angle $\gamma$, satisfying inequality

$$
\cos \gamma>\frac{A_{1}(\Delta p)^{2}}{2 p_{1}\left(\left(p_{0}+p_{1}\right) A_{01}-p_{1} A_{0}-p_{0} A_{1}-\sqrt{G E}\right)},
$$

problem (10)-(9) has no solutions. 
Let $D_{0}$ be the the disk, which radius $r$ satisfies the inequality (17). Then

$$
\begin{gathered}
A_{0}=\pi r^{2}, \quad p_{0}=2 \pi r, \quad 2 A_{01}=r p_{1}, \\
G=\pi r^{2}\left(p_{1}^{2}-4 \pi A_{1}\right), \quad E=r p_{1}-\pi r^{2}-A_{1} .
\end{gathered}
$$

Note that the second of inequalities (22) holds because of (17) and the first one holds automatically. Substituting last formulas in inequality (24) we obtain Theorem 11 after algebraic transformations.

Example. Let domain $D_{1}$ be the regular $n$-polygon circumscribed around a circle of radius $r$. Then the inequality (19) takes the form

$$
\cos \gamma>\frac{1}{2}\left(1+\sqrt{\frac{\pi}{n \tan \frac{\pi}{n}}}\right) .
$$

For large $n$ we can write more simple formula

$$
\gamma<\frac{\pi}{n \sqrt{6}} \text {. }
$$

We see that this estimate is weaker than the exact one ([3], Th. 6.2): $\gamma<\pi / n$, but it holds the same form in case of smoothed angles.

\section{Appendix.}

Let us consider now the general steps for proof Theorem 1. Hereafter we denote by $C$ (with subscripts or without them) the constants depending on geometrical characteristics of domain $D$.

It is evident that in convex domain $D$ Poincaré inequality holds

$$
\iint_{D} u^{2} d x d y \leq \frac{1}{A}\left(\iint_{D} u d x d y\right)^{2}+\mu \iint_{D}|\nabla u|^{2} d x d y .
$$

Let

$$
v=u-\frac{R p}{k A}
$$

Function $v$ satisfies the equation

$$
\Delta v=k v+\frac{R p}{A}
$$

and $v_{n}=R$ on $\Gamma$. Let us integrate (2) over $D$. It is easy to see that

$$
\iint_{D} v d x d y=0
$$

We subtract Equation (3) from Equation (25). We obtain

$$
\Delta(v-z)=k(v-z)+k z .
$$

Denote $\omega=v-z$. Then

$$
\Delta \omega=k \omega+k z
$$


We multiply (26) on $\omega$ and integrate over $D$. We obtain

$$
\iint_{D} \Delta \omega \omega d x d y=\iint_{D} k \omega^{2} d x d y+\iint_{D} z \omega d x d y .
$$

We transform the left side of (27) by well-known formulas, taking into account that $\omega_{n}=0$. We obtain

$$
-\iint_{D}|\nabla \omega|^{2} d x d y=\iint_{D} k \omega^{2} d x d y+\iint_{D} k z \omega d x d y .
$$

Taking into account that

$$
\iint_{D} \omega d x d y=0
$$

we use the Poincaré inequality

$$
\begin{aligned}
\left(k+\frac{1}{\mu}\right)\|\omega\|_{L_{2}}^{2} & \leq k\|\omega\|_{L_{2}}^{2}+\iint_{D}|\nabla \omega|^{2} d x d y \\
& \leq k \iint_{D} \omega^{2} d x d y+\iint_{D}|\nabla \omega|^{2} d x d y,
\end{aligned}
$$

and the Cauchy-Schvarz-Bunyakovskii inequality

$$
-k \iint_{D} \omega z d x d y \leq|k|\left(\iint_{D} z^{2} d x d y\right)^{1 / 2}\left(\iint_{D} \omega^{2} d x d y\right)^{1 / 2} .
$$

We obtain after algebraic transformations

$$
\|\omega\|_{L_{2}} \leq \frac{\mu|k|}{1+\mu k}\|z\|_{L_{2}}
$$

By S.L. Sobolev embedding theorem:

$$
\left(\max _{\bar{D}} \omega\right)^{2} \leq C_{1}\left\|\nabla_{2} \omega\right\|_{L_{2}}^{2}+C_{2}\|\omega\|_{L_{2}}^{2}
$$

$L_{2}$ - norm of second derivitives of $\omega$ in plane convex domain is estimated from $L_{2}$ - norm of operator $\Delta \omega$. The detailed proof of this estimate for solution of the first boundary value problem is given in [10]. The same proof yields the same estimate for solutions of second boundary value problem as well. Indeed, let $g=\omega_{x x} \omega_{y y}-\omega_{x y}^{2}$. It is evident that

$$
\omega_{x x}^{2}+2 \omega_{x y}^{2}+\omega_{y y}^{2}=(\Delta \omega)^{2}-2 g .
$$


Using the identity

$$
2 \iint_{D} g d x d y=\int_{\Gamma}\left(\omega_{x} \omega_{x y}-\omega_{y} \omega_{x x}\right) d x-\left(\omega_{x} \omega_{y y}-\omega_{y} \omega_{x y}\right) d y
$$

we obtain because of boundary condition of problem (2) and convexity of domain $D$

$$
2 \iint_{D} g d x d y=\int_{\Gamma} K|\nabla \omega|^{2} d s>0
$$

and the estimate is proved.

Furthermore, we obtain from (26)

$$
\left\|\nabla_{2} \omega\right\|_{L_{2}}^{2} \leq\|\Delta \omega\|_{L_{2}}^{2} \leq 2 k^{2}\left(\|\omega\|_{L_{2}}^{2}+\|z\|_{L_{2}}^{2}\right) .
$$

The statement of Theorem 1 follows from the substitution of the latter estimate in (30) using (29).

Remark. Analyzing the proof of Theorem 1, we can see that requirement to convexity of domain $D$ is excessive. Indeed, we can require only the realizability of the Poincaré inequality, S.L. Sobolev embedding theorem and the possibility to estimate $\left\|\nabla_{2} u\right\|_{L_{2}}$ by means of $\|\Delta u\|_{L_{2}}$. These conditions are contained in $[\mathbf{1 0}]$.

Acknowledgments. We would like to thank Professor R. Finn for his comments and suggestions. Professor R. Finn has played a critical role in the design and the completion of this paper. We are grateful to Doctor G.N. Krichevets for helpful discussion.

\section{References}

[1] W. Blaschke, Kreis und Kugel, Walter de Gruiter \& Co, Berlin, 1956, MR 17,1123d, Zbl 070.17501.

[2] P. Concus and R. Finn, On capillary free surfaces in the absence of gravity, Acta Math., 132 (1974), 177-198, MR 58 \#32327a, Zbl 382.76003.

[3] R. Finn, Equilibrium Capillary Surfaces, Springer-Verlag, 1986, MR 88f:49001, Zbl 583.35002.

[4] E. Giusti, On the equation of surfaces of prescribed mean curvature: Existence and uniqueness without boundary conditions, Invent. Math., 46 (1978), 111-137, MR 58 \#7337, Zbl 381.35035.

[5] G. Keady and A. McNabb, Functions with constant Laplacian satisfying homogeneous Robin boundary conditions, IMA J. of Applied Math., 50 (1993), 205-224, MR 94g:35064, Zbl 803.35025.

[6] A.A. Kosmodem'yanskii Jr., On the comparison theorem of second boundary value problem for Helmholtz equation, in 'PDE Prague '98, Book of Abstracts', Praha, Karolinium nakladatelstvi univerzity Karlovy, 72-73. 
[7] _ A theorem on representation of the solution of the second boundary value problem, Soviet Math. Dokl., 43(2) (1991), 371-373.

[8] _ The third boundary value problem for the Poisson equation, Mathematical Notes, 63(2) (1998), 257-260, Zbl 915.35019.

[9] _ The comparisson of capillary surfaces heights in case of small gravity, Nonlinear Analysis, 43 (2001), 937-942.

[10] O.A. Ladyzhenskaya and N.N. Ural'tseva, Linear and Quasilinear Equations of Elliptic Type, Nauka, Moscow, 1973, MR 58 \#23009.

[11] L.A. Ljusternik, Convex Figures and Polyhedra, GITTL, Moscow, 1956, MR 36 \#4435.

[12] D. Siegel, Height estimates for capillary surfaces, Pacific J. Math., 88 (1980), 477-516, MR 82h:35037, Zbl 453.35030.

[13] _ The behavior of a capillary surface for small Bond number, in "Variational methods for free surface interface", eds. P. Concus and R. Finn, Springer-Verlag, (1987), 109-113, MR 8f:53010.

Received March 19, 1999 and revised June 19, 2000.

Moscow State University of Railways (MIIT)

Dept. of Computational Mathematics

Ul. OBRAZTSOVA 15

Moscow

Russia

E-mail address: mitja@farber.mccme.ru 